\title{
Detection of blood spots in eggs by hyperspectral transmittance imaging
}

\author{
Zhe Feng, Chengqiao Ding, Weihao Li, Di Cui* \\ (College of Biosystems Engineering and Food Science, Zhejiang University, Hangzhou 310058, China)
}

\begin{abstract}
Blood spots are one of undesired inclusions in eggs, whose detection success is highly dependent on shell color. This research reports a method for detecting blood spots in light brown-shelled eggs on the basis of hyperspectral transmittance images. The normalized spectra of intact eggs and their shells were acquired. Five feature wavelengths of intact eggs selected by the successive projections algorithm and 3 absorption peak locations of eggshells were regarded as characteristic bands. The k-nearest neighbor $(\mathrm{kNN})$ and support vector machine (SVM) algorithms were adopted to develop detection models. The latter achieved better performance. The overall classification accuracy increased to $100 \%$ by merging normalized spectra of intact eggs at 5 feature wavelengths with 3 absorption peaks of eggshells as input variables of SVM-based model. Moreover, a practical SVM-based model with $96.43 \%$ overall classification accuracy was established by replacing inputs with normalized spectra of intact eggs at characteristic bands.
\end{abstract}

Keywords: hyperspectral transmittance imaging, non-destructive detection, blood-spot, egg

DOI: $10.25165 /$ j.ijabe.20191206.5376

Citation: Feng Z, Ding C Q, Li W H, Cui D. Detection of blood spots in eggs by hyperspectral transmittance imaging. Int J Agric \& Biol Eng, 2019; 12(6): 209-214.

\section{Introduction}

Blood spots appearing on the yolk or in the albumen will affect the quality and grade of eggs. Different countries have different standards for grading eggs. The USDA grades for shell eggs clearly stipulate that small blood spots aggregating not more than $1 / 8$ inch $(3.2 \mathrm{~mm})$ in diameter may be classified as B quality ${ }^{[1]}$. China's Ministry of Commerce promulgated that eggs of Grade AA, A and B shouldn't contain blood spots, meat spots and other foreign matters in the industry standards for fresh egg grading in $2011^{[2]}$. Therefore, the present detection of blood spots is important for egg grading.

Traditionally, egg internal defect is examined by candling, which is a process that passes the egg over a bright light to make its interior visible. The determination will be made by well-trained workers based on their observation results. Since this method is labor intensive and heavily depending on the experience of the workers, the traditional method is hard to meet the demand of high accuracy and throughput. In recent years, many advanced technologies were employed to develop objective methods for egg internal defect detection, in which imaging and spectroscopy attracted great attention of researchers ${ }^{[3]}$. Patel et al. ${ }^{[4]}$ used a machine vision system to acquire the gray images of blood spot eggs and trained the neural network model for blood spot detection by the histograms generated from the images. The detection accuracy of blood spotted eggs was $86.7 \%$. Then Patel et al. ${ }^{[5]}$ improved this method by using a color imaging system. The accuracy of the neural network model trained by histograms of red,

Received date: 2019-09-02 Accepted date: 2019-11-10

Biographies: Zhe Feng, $\mathrm{PhD}$ candidate, research interest: Nondestructive inspection of Agro-products, Email: 11713010@zju.edu.cn; Chengqiao Ding, $\mathrm{PhD}$ candidate, research interest: Nondestructive inspection of Agro-products, Email: 21613010@zju.edu.cn; Weihao Li, Master candidate, research interest: Nondestructive inspection of Agro-products, Email: 21713017@zju.edu.cn

*Corresponding author: Di Cui, $\mathrm{PhD}$, Associate Professor, research interest: Sensing technology for Agro-product quality, Mailing address: 866 Yuhangtang Rd., Hangzhou 310058, Zhejiang, China. Tel: +86-571-889-828-20, Email: dicui@zju.edu.cn. green and blue component images increased to $92.8 \%$. Nakano et al. ${ }^{[6]}$ also developed a color imaging system to obtain the transmission images of eggs by candling. The normalized chromatic component $g$ was introduced to detect the bloody eggs from the normal eggs. When the threshold of $g$ was set to 2.9, a total detection accuracy of $94.2 \%$ for the white-shelled bloody eggs was acquired. Besides the imaging technology, the spectroscopy technology was also used for detecting blood pots in eggs. Usui et al. ${ }^{[7]}$ applied the near infrared spectroscopy to detect blood spots in white-shelled eggs, established detection model by the partial least square (PLS) method and achieved $96.8 \%$ accuracy for blood-spot eggs. Compared to white-shelled eggs with blood spots, brown-shelled eggs with blood spots are more difficult to be detected since the images of the brown shelled eggs were quite similar to those of the blood spots in the white shelled eggs ${ }^{[8]}$. Xu et al. ${ }^{[9]}$ analyzed visible spectroscopy of brown-shelled eggs by the least squares support vector machines (LS-SVM) method and acquired $91.7 \%$ accuracy for blood-spot detection. Gielen et al. ${ }^{[8]}$ found that the absorption peak of the pigment protoporphyrin in brown-shelled eggs was at $589 \mathrm{~nm}$, while that of hemoglobin in blood spots was at $577 \mathrm{~nm}$. Their absorption peak locations were very close, which resulted in the relative lower detection accuracy for brown-shelled eggs with blood spots. In order to achieve higher accuracy, the influence of the protoporphyrin needs to be compensated.

Hyperspectral imaging technology combines conventional imaging and spectroscopy techniques ${ }^{[10]}$ and has shown great potential in detection of egg internal quality. Zhang et al. used hyperspectral imaging with multivariate analysis to detect the freshness, bubble formation and scattered yolk of white-shelled eggs ${ }^{[11]}$. Smith et al. ${ }^{[12]}$ measured a total of 240 white-shell table eggs by hyperspectral imaging in transmittance mode and a ratio of images at $577 \mathrm{~nm}$ and $565 \mathrm{~nm}$ were used to determine if pixels from the image were positive for blood. A greater than $90 \%$ accuracy rate was obtained. To the best of authors' knowledge, there has been no report on detecting blood spots in brown-shelled eggs by hyperspectral imaging.

The overall objective of this study was to investigate the 
possibility of detecting blood spot in light brown-shelled eggs by hyperspectral transmittance imaging. The specific objectives of the study were (a) to select feature wavelengths from hyperspectral transmittance image data; (b) to establish qualitative detection models; and (c) to compensate the influence of the protoporphyrin in eggshells on the classification accuracies of detection models.

\section{Materials and methods}

\subsection{Sample preparation}

A total of 98 light brown-shelled eggs were bought from a local mart and stored in a refrigerator at $4^{\circ} \mathrm{C}$. All the eggs were moved to the experimental environment 4 hours ahead of measurement to allow them to reach room temperature. Then they were divided into 2 groups, normal group (50 eggs) and defect group (48 eggs). In the normal group, 36 samples were randomly selected for model calibration and the rest were for model validation. In previous studies, blood spot eggs were made by injecting $0.03-0.05 \mathrm{~mL}$ blood into normal eggs ${ }^{[9,13,14]}$. In this research, the defect samples were prepared by injecting $0.04 \mathrm{~mL}$ chicken blood mixture (1:3 mixture of $10 \%$ transparent sodium citrate solution and fresh chicken blood) in their albumen. The sodium citrate can prevent blood clotting, which makes the injection easier. Since the absorption spectrum of sodium citrate solution does not show distinct absorption peak at 500-700 nm, its addition has little influence on the spectra of defect samples at 500-700 $\mathrm{nm}^{[15]}$. In the defect group, 34 samples were also randomly selected for model calibration and the others were for model validation.

\subsection{Data acquisition}

The hyperspectral transmittance image (HTI) acquisition system was shown in Figure 1, which consisted of an array charge coupled device (CCD) camera (C8484-05G01, Hamamatsu Photonics, Japan) and a line scanning spectrograph (ImSpector V10E-QE, Spectral Imaging Ltd., Oulu, Finland) ranging from 450 to $1000 \mathrm{~nm}$ with a spectral resolution of $2.8 \mathrm{~nm}$. A 150 watt halogen fiberoptic light source (2900, Illumination Technologies, Inc., USA) with a $96.5 \mathrm{~mm}$ single lightguide (9133HT, Illumination Technologies, Inc., USA) was used for illumination. An electric-driving displacement platform (TSA200-B, Zolix Instruments Co., Ltd., Beijing, China) with a stage was managed by a controller to realize line scan. The stage with a $\varphi 25 \mathrm{~mm}$ central aperture was used for vertically placing eggs with their big end up, which was made of aluminum oxide and had matte black anodized finish surface to reduce unwanted reflection. Four stainless steel posts fixed on the platform were used to support the stage and provide installation space for the lightguide. The whole system was installed in a black chamber to avoid ambient light. Moreover, a computer (Inter RcoreTM2 4400@2.00 GHz, ACER, China) supervised the entire procedure of HTI acquisition.

After measuring the HTI of each intact egg, the egg was broken from the small end for visual inspection of its internal quality. The shell of big end was collected and cleaned with deionized water. Its membrane was removed by manual. After a further $24 \mathrm{~h}$ of air-drying, its HTI was measured. For each sample, HTIs of intact eggs and shell fragment were both measured.

\subsection{Data processing and analysis}

The whole egg area was as the region of interest (ROI) from the intact egg HTIs, while the ROI of shell fragment was extracted manually. Figure 2 shows the technical flowchart of the spectral data analysis. During the whole processing, the mean spectra extracted from raw HTI of ROI was firstly corrected and normalized to acquire normalized spectra, which could minimize the influence of transmitted light intensity caused by eggshell thickness. Then the feature wavelengths of intact eggs were selected by successive projections algorithm to reduce information redundancy. Besides, the absorption peak locations of eggshell fragments were also extracted. At last, 4 groups of input variables based on above analysis were put into two classifiers of SVM and $\mathrm{kNN}$ to establish detection models for blood spots. The details of data processing and analysis were described at the following sections.

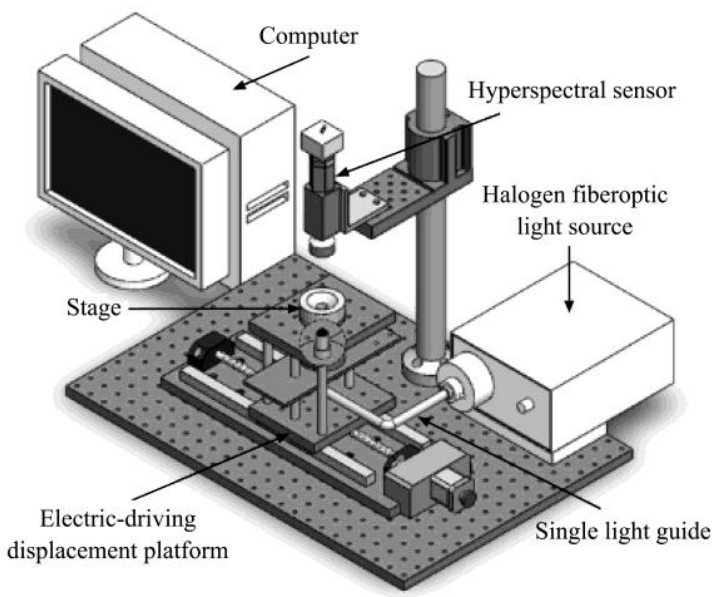

Figure 1 Hyperspectral imaging system in transmittance mode for eggs
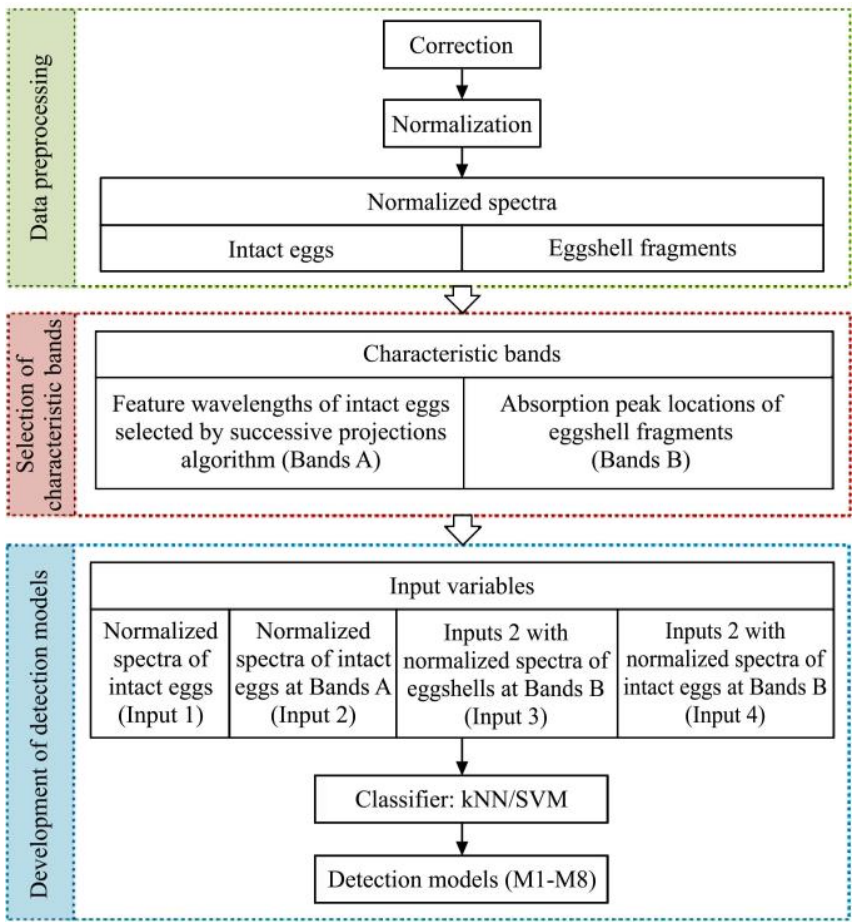

Figure 2 Flowchart of data processing and analysis

\subsubsection{Data preprocessing}

In order to acquire relative HTIs, raw data must be corrected using the following equation ${ }^{[16]}$ :

$$
T_{R}=\frac{T_{S}-D}{T_{T}-D}
$$

where, $T_{R}$ is the relative HTI; $T_{S}$ and $T_{T}$ are the raw HTIs of the sample and the reference; and $D$ is the dark image. In this study, the Teflon panels with $25 \mathrm{~mm}$ and $15 \mathrm{~mm}$ thickness were used on the stage in Figure 1 as the reference for intact egg and shell fragment, respectively. 
After the correction, the mean relative transmittance spectra (RTS) of both intact eggs and shell fragments were extracted from their relative HTI. And the spectral range was narrowed to $500-700 \mathrm{~nm}$ for minimizing the noise resulting from sodium citrate solution, and then normalized to [0,1] using Equation (2). The normalized spectra were used for the following data analysis. All the data preprocessing and analysis were performed in Matlab.

$$
X_{i}^{\prime}=\frac{X_{i}-X_{\min }}{X_{\max }-X_{\min }}
$$

where, $X_{i}^{\prime}$ is the normalized spectral value at the wavelength $i ; X_{i}$ is the raw spectral value at the wavelength $i ; X_{\min }$ and $X_{\max }$ are the minimum and maximum values of $X_{i}$, respectively.

\subsubsection{Selection of characteristic bands}

The high information redundancy in hyperspectral image data will lead to convergence instability in classification models, which can be reduced by selection of the most informative spectral bands. The successive projections algorithm (SPA) uses a simple projection operation in vector space to obtain a subset of variables with small collinearity ${ }^{[17]}$. It is a forward variable selection algorithm for multivariate calibration. SPA's variable selection principle is that the selected new variable is among all remaining variables. One of which has the largest projection value on the orthogonal subspace of the previously selected variable ${ }^{[18]}$. This method begins with one wavelength and calculates the projection on the unselected wavelength for each iteration and introduces the wavelength of the largest projection vector into the wavelength combination until it is cycled $N$ times. Detailed instruction of SPA see Ref. ${ }^{[17]}$. In this research, the SPA was employed to select feature wavelengths from normalized spectra of intact eggs (denoted as Bands A). Besides, the absorption peak locations of eggshells were regarded as another group of characteristic bands (denoted as Bands B).

\subsubsection{Development of detection models}

The $k$-nearest neighbor $(\mathrm{kNN})$ algorithm is a non-parametric method, which is sensitive to the local structure of the data ${ }^{[19]}$. The choice of $k$ has a great influence for classification. One popular way for choosing the empirically optimal $k$ is via bootstrap $\operatorname{method}^{[20]}$. The support vector machines (SVM) is another supervised learning algorithm for linear and non-linear classification, which consists in finding the optimum separating hyperplan between classes due to the identification of the most representative training samples ${ }^{[21]}$. It is widely used to analysis multispectral and hyperspectral images and shows many advantages in solving small samples, non-linear and high dimensional pattern recognition ${ }^{[22]}$. In this research, both $\mathrm{kNN}$ and SVM algorithms were adopted to establish the detection models with different inputs.

Firstly, the kNN- and SVM-based models for blood spots detection were created on the basis of the normalized spectra and selected feature wavelengths of intact eggs by SPA (denoted as M1-M4), respectively. Subsequently, in order to reduce the influence of the pigment protoporphyrin in light-brown eggshells on the detection accuracy of blood spots, normalized spectra of intact eggs at the feature wavelengths (Bands A) were merged with the absorption peaks of eggshells (Bands B) to establish accurate detection models by means of kNN and SVM algorithms (M5 and M6). Since nondestructive measurement of eggshell spectra is impossible at present, it is necessary to find a method to take place of eggshell spectra for model development. Therefore, the normalized spectra of intact eggs at the same wavelengths as the absorption peak locations of eggshell spectra served as the inputs of kNN- and SVM-based practical detection models (M7 and M8). The performances of these models were compared and evaluated by their classification accuracies. Good models have higher classification accuracies.

\section{Results and discussions}

\subsection{Color information}

The Red Green Blue (RGB) images were formed from HTIs of intact eggs at $661 \mathrm{~nm}$ (Red), $554 \mathrm{~nm}$ (Green), $450 \mathrm{~nm}$ (Blue) wavelength. Then, The RGB values were converted to $L^{*} a * b *$ values (Table 1) by Matlab 7.14.0 software (The Math Works, Inc., Natick, Mass., USA). In Table 1 , the values of $b^{*}$ were all positive, which showed the realistic egg color were yellowish. Besides, the color of the egg was mostly reddish. It illustrated that the egg samples were brownish.

Table 1 Color information of egg samples

\begin{tabular}{cccc}
\hline Color Parameter & $L^{*}$ & $a^{*}$ & $b^{*}$ \\
\hline Maximum & 62.24 & 50.43 & 62.03 \\
Minimum & 28.85 & -1.52 & 33.29 \\
Standard deviation & 5.51 & 10.24 & 5.86 \\
\hline
\end{tabular}

\subsection{Spectra interpretation}

The mean RTS of normal (50) and defect (48) samples with standard deviation (SD) were shown in Figure $3 \mathrm{a}$. In order to avoid the impact of the sodium citrate solution on blood spots detection, the RTS of all samples in the range of 500-700 nm were extracted and normalized to $[0,1]$ using equation (2) (Figure $3 b$ ). The mean RTS of defect (48) samples were similar to those of normal ones. The relative transmittance of all light brown-shelled eggs gradually increased with wavelength in the range of 500$700 \mathrm{~nm}$. Intact eggs presented one distinct absorption peak at $642.72 \mathrm{~nm}$ and two flat terraces at 500-540 nm and 574-585 nm.

The data process for eggshells was the same as that for intact eggs. The mean RTS of eggshells with standard deviation were displayed in Figure 4a. The normalized spectra in the range of 500-700 nm of eggshells with standard deviation were shown in Figure $4 \mathrm{~b}$. Three absorption peaks of light brown-shelled eggshells located at 539.3, 590.1 and $644.0 \mathrm{~nm}$ were observed, which was due to the pigment protoporphyrin in the shell. Similar results were also obtained by other researchers for the brown shelled eggs ${ }^{[8]}$.

Comparing the results shown in Figure $3 \mathrm{a}$ with $4 \mathrm{a}$, it was found that intact eggs had significant difference with eggshells in the SW-NIR range. This was due to that eggs contained a lot of protein while eggshells mainly contained calcium carbonate. Relative to normalized spectra of eggshells shown in Figure $4 \mathrm{~b}$, normalized spectra of intact eggs showed only one pronounced absorption peak at about $642 \mathrm{~nm}$. It meant that intact egg with light brown shell didn't present the peaks at around 539 and $590 \mathrm{~nm}$. In other studies, white eggs had no absorption peak in the range of $500-700 \mathrm{~nm}$, but brown-shelled eggs $\operatorname{did}^{[8]}$. Therefore, the number of absorption peaks of intact egg might be caused by its shell color. The absorption peaks might become more and more distinct with the increasing of shell color.

\subsection{Feature extraction}

It was important to reduce the redundant formation in normalized spectra of intact eggs. In this study, the 5 feature wavelengths were extracted by SPA from normalized spectra of intact eggs at 500-700 nm, which were 549.16, 564.01, 578.91, 597.6 and $633.91 \mathrm{~nm}$ (Figure 5). The selected wavelengths 
contained the characteristic absorption band of hemoglobin in blood spots at $578.91 \mathrm{~nm}$. Other characteristic bands were located

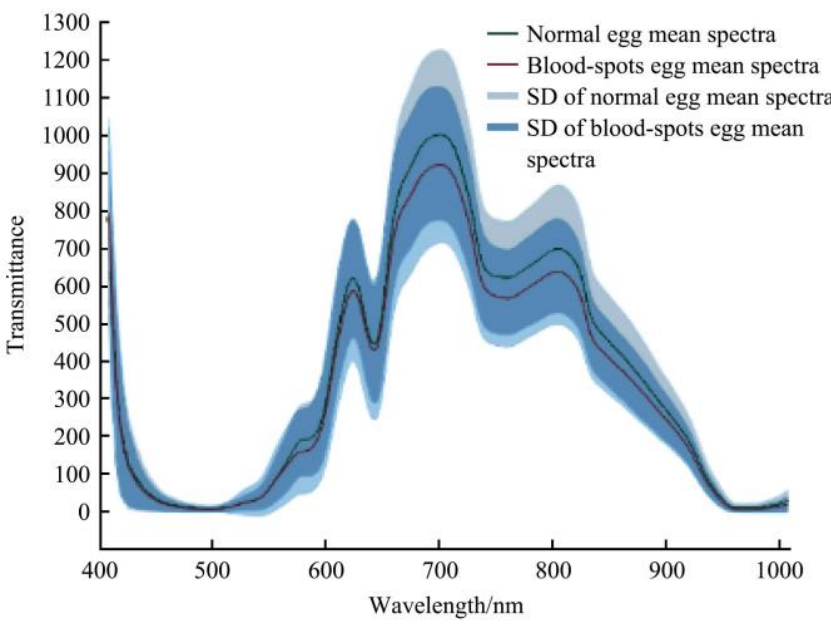

in the range of $540-640 \mathrm{~nm}$ and reported to have the potential of predicting egg freshness ${ }^{[23]}$.

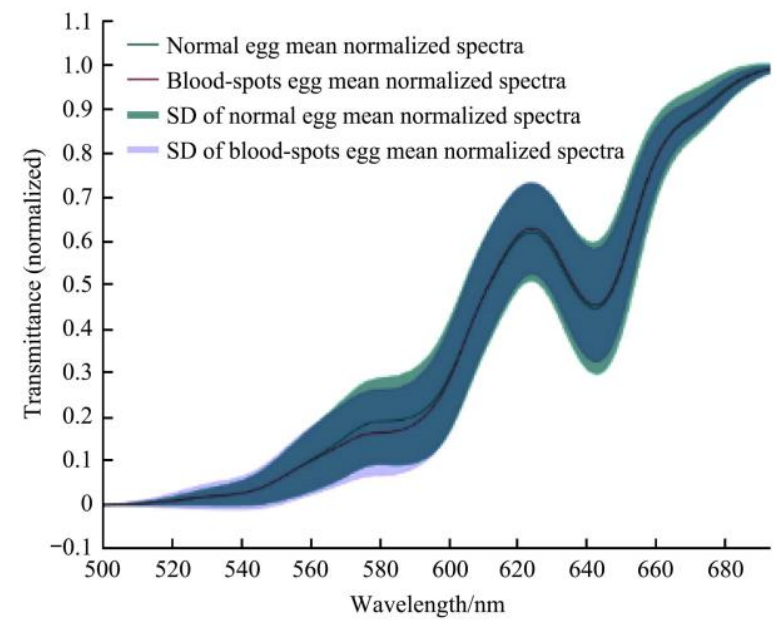

b.

Figure 3 Relative transmittance spectra (RTS) (a) and normalized spectra of intact eggs with and without blood spots (b) (SD: Standard deviation)

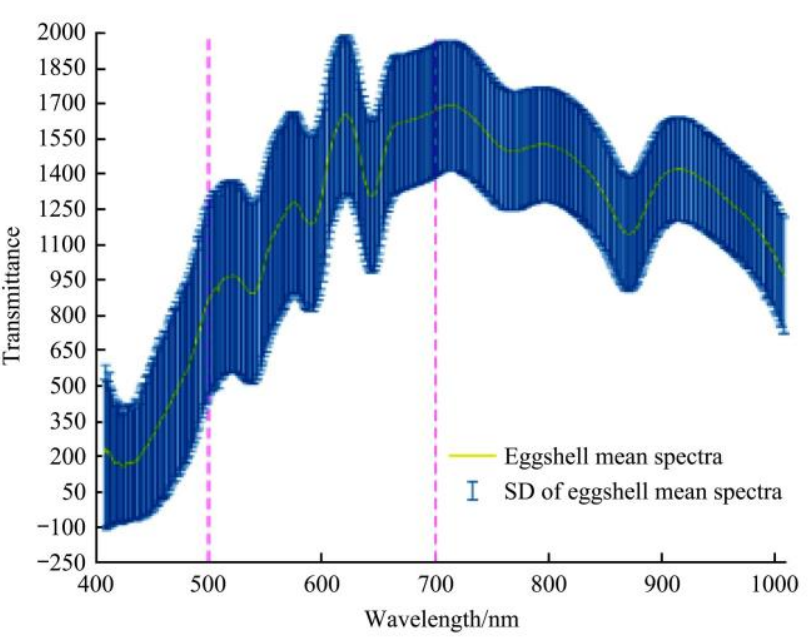

a.

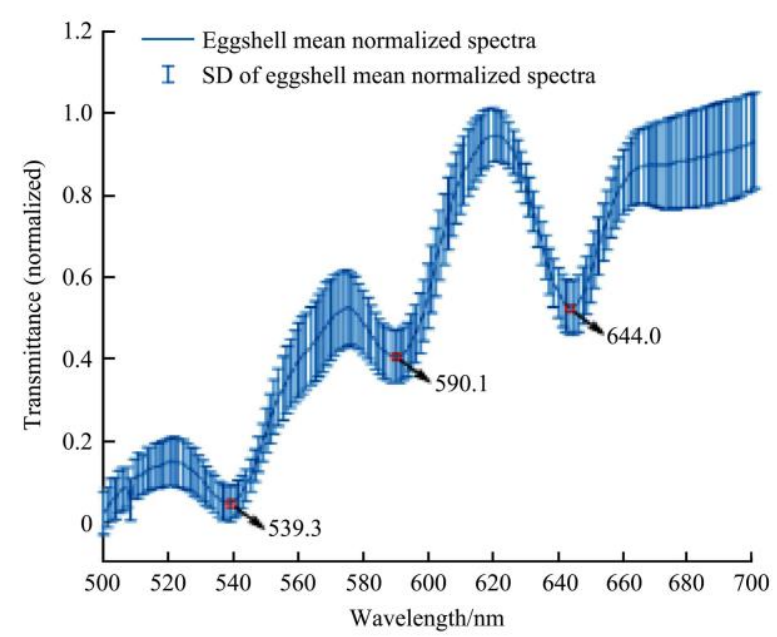

b.

Note: SD: Standard deviation.

Figure 4 Relative transmittance spectra (RTS) (a) and normalized spectra of eggshells (b)

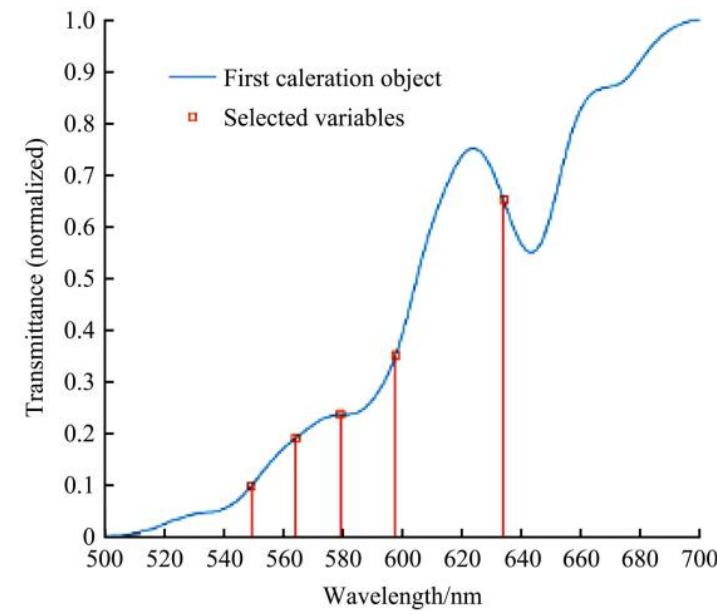

Figure 5 The 5 feature wavelengths extracted by successive projections algorithm (SPA) from normalized spectra of intact eggs

\subsection{Blood spot detection models}

The results for blood-spot detection models developed by kNN and SVM algorithms with different input data were shown in Table 2 and 3. By comparing these tables, it was indicated that the
SVM-based models achieved better performance than the kNN-based models. The classification accuracies of kNN-based models (M1, M3, M5 and M7) had similar changing trend with those of SVM-based models (M2, M4, M6 and M8). In the case of SVM-based models, $100 \%$ overall classification accuracy of model M6 were achieved by using normalized spectra of intact eggs at the 5 feature wavelengths coupled with the 3 absorption peaks of eggshells as input variables (Inputs 3). When the input variables only included normalized spectra of intact eggs at the 5 feature wavelengths (Inputs 2), the overall classification accuracy of model M4 reduced to $85.71 \%$. Moreover, when the inputs were changed into whole normalized spectra of intact eggs (Inputs 1), the classification accuracy of models M2 were $96.43 \%$, which was still lower than that of model M6 and cost more computing time. Above results also confirmed that the influence of the protoporphyrin in eggshells on the classification accuracies of detecting blood spots could be compensated by adding normalized spectra of eggshells to input variables of detection models. However, it was impossible to nondestructively acquire spectra of eggshells in practical production. Therefore, Inputs 2 with normalized spectra of intact egg at the same wavelengths as the 
absorption peak locations of eggshells served as input variables for developing practical detection model M8, whose overall classification accuracy degraded from $100.00 \%$ to $96.43 \%$. However, this result was still better than that of model M4 only using normalized spectra of intact eggs at the 5 feature wavelengths. For kNN-based models, the highest classification accuracy of $82.14 \%$ was achieved by using either Inputs 1 or Inputs 3, while the lowest classification accuracy of $71.43 \%$ were obtained by using Inputs 2.

Table 2 Classification results for the validation set by k-nearest neighbor $(\mathrm{kNN})$ algorithm with different inputs

\begin{tabular}{|c|c|c|c|c|c|c|}
\hline \multirow{2}{*}{ Model } & \multirow{2}{*}{$\begin{array}{c}\text { Input } \\
\text { variables }\end{array}$} & \multirow{2}{*}{$\begin{array}{c}\text { Number of } \\
\text { inputs }\end{array}$} & \multicolumn{2}{|c|}{$\begin{array}{c}\text { Number of misclassified } \\
\text { eggs }\end{array}$} & \multirow{2}{*}{$\begin{array}{c}\text { Overall } \\
\text { classification } \\
\text { accuracy }\end{array}$} & \multirow{2}{*}{$\begin{array}{c}k \\
\text { value }\end{array}$} \\
\hline & & & $\begin{array}{l}\text { Normal } \\
\text { (total:14) }\end{array}$ & $\begin{array}{l}\text { Defect } \\
\text { (total:14) }\end{array}$ & & \\
\hline M1 & Inputs $1^{\mathrm{a}}$ & 162 & 5 & 0 & $82.14 \%$ & 4 \\
\hline M3 & Inputs $2^{\mathrm{b}}$ & 5 & 3 & 5 & $71.43 \%$ & 1 \\
\hline M5 & Inputs $3^{\mathrm{c}}$ & 8 & 1 & 4 & $82.14 \%$ & 9 \\
\hline M7 & Inputs $4^{\mathrm{d}}$ & 8 & 1 & 5 & $78.57 \%$ & 1 \\
\hline
\end{tabular}

Note: ${ }^{a}$ Inputs 1: whole normalized spectra of intact eggs at $500-700 \mathrm{~nm}$.

${ }^{\mathrm{b}}$ Inputs 2: normalized spectra of intact eggs at the feature wavelengths selected by SPA.

${ }^{\mathrm{c}}$ Inputs 3: Inputs 2 with the absorption peaks of eggshells (Bands B).

${ }^{\mathrm{d}}$ Inputs 4: Inputs 2 with normalized spectra of intact eggs at Bands B.

Table 3 Classification results for the validation set by support vector machines (SVM) algorithm with different inputs

\begin{tabular}{cccccc}
\hline & & & \multicolumn{2}{c}{ Number of misclassified eggs } & Overall \\
\cline { 5 - 6 } Model & $\begin{array}{c}\text { Input } \\
\text { variables }\end{array}$ & $\begin{array}{c}\text { Number } \\
\text { of inputs }\end{array}$ & $\begin{array}{c}\text { Normal } \\
\text { (total:14) }\end{array}$ & $\begin{array}{c}\text { Defect } \\
\text { (total:14) }\end{array}$ & accuracy \\
\hline M2 & Inputs 1 $^{\mathrm{a}}$ & 162 & 0 & 1 & $96.43 \%$ \\
M4 & Inputs 2 $^{\mathrm{b}}$ & 5 & 0 & 4 & $85.71 \%$ \\
M6 & Inputs 3 $^{\mathrm{c}}$ & 8 & 0 & 0 & $100.00 \%$ \\
M8 & Inputs 4 $^{\mathrm{d}}$ & 8 & 0 & 1 & $96.43 \%$ \\
\hline
\end{tabular}

Note: ${ }^{a}$ Inputs 1: whole normalized spectra of intact eggs at $500-700 \mathrm{~nm}$.

${ }^{\mathrm{b}}$ Inputs 2: normalized spectra of intact eggs at the feature wavelengths selected by SPA.

${ }^{\mathrm{c}}$ Inputs 3: Inputs 2 with the absorption peaks of eggshells (Bands B).

${ }^{\mathrm{d}}$ Inputs 4: Inputs 2 with normalized spectra of intact eggs at Bands B.

\subsection{Discussion}

The mean RTS of eggshells had significant difference with intact eggs in the SW-NIR range. There were three absorption peaks of light brown-shelled eggshells, which was due to the pigment protoporphyrin. Taking this into consideration could help improve the predictive power of the model. It could be found in this research that model M6 merged normalized spectra of intact eggs at the 5 feature wavelengths with the 3 absorption peaks of eggshells as inputs had the best result compared with other models. However, it was impossible to nondestructively acquire spectra of eggshells. For the purpose of nondestructive detection, the absorption peaks of eggshells were replaced by normalized spectra of intact egg at the same wavelengths as input variables for developing practical detection models. The accuracy of model M8 was $96.43 \%$ which was same as Model M2. Whereas, model M8 had fewer input variables and less computing time, which meant that model M8 had certain application value.

\section{Conclusions}

This preliminary results obtained from this research demonstrates that this hyperspectral transmittance imaging method could detect blood spots in light brown-shelled eggs.

(1) This research found 8 feature wavelengths to establish the model. Among these wavelengths, there were three absorption peaks located at 539.3, 590.1 and $644.0 \mathrm{~nm}$ that were observed from normalized spectra of light brown-shelled eggshells. Besides, the 5 feature wavelengths were extracted by SPA from normalized spectra of intact eggs at 500-700 nm, which were 549.16, 564.01, 578.91, 597.6 and $633.91 \mathrm{~nm}$. Among them, the wavelength $578.91 \mathrm{~nm}$ was the characteristic absorption band of hemoglobin in blood spots.

(2) This research established 8 models by kNN and SVM. Relative to kNN-based model, the SVM-based model yielded better performance. The accuracy of SVM-based model was $100 \%$ and $96.43 \%$, which used normalized spectra of intact eggs at the 5 feature wavelengths with the 3 absorption peaks of eggshells and 8 feature wavelengths of intact eggs as inputs respectively.

This paper reports the preliminary results in detecting blood spots in light brown-shelled eggs by hyperspectral transmittance imaging. More comprehensive studies are necessary to verify and/or improve the developed methods for different amount of blood in light brown-shelled eggs.

\section{Acknowledgements}

The authors gratefully acknowledge the support of this program by the National Natural Science Foundation of China (Grant No. 31571764) and the National Key Research and Development Program of China (2017YFC1600805). Any opinions, findings, and conclusions expressed in this publication are those of the authors and do not necessarily reflect the views of Zhejiang University. The trade and manufacturers' names are necessary to report factually on the available data.

\section{[References]}

[1] USDA. United States Standards: Grades, and weight classes for shell eggs. in: AMS-56, 2000.

[2] MOFCOM, Grading of shell hen eggs and duck eggs. SB/T 10638-2011, China, 2011.

[3] Brant A W, Norris K H, Chin G. A spectrophotometric method for detecting blood in white-shell eggs. Poultry Science, 1953; 32(2): 357-363.

[4] Patel V C, Mcclendon R W, Goodrum J W. Detection of blood spots and dirt stains in eggs using computer vision and neural networks. Applied Engineering in Agriculture, 1996; 12(2): 253-258.

[5] Patel V C, Mcclendon R W, Goodrum J W. Color computer vision and artificial neural networks for the detection of defects in poultry eggs. in: Panigrahi S, Ting K C (Eds.) Artificial intelligence for biology and agriculture. Springer Netherlands, Dordrecht, 1998; pp. 163-176.

[6] Nakano K, Sasaoka K, Ohtsuka Y. A study on non-destructive detection of abnormal eggs by using image processing. 2nd Asian Conference for Information Technology in Agric, Asian Federation for Information Technology in Agriculture, 2000; pp. 345-352.

[7] Usui Y, Nakano K, Motonaga Y. A study of the development of non-destructive detection system for abnormal eggs. EFITA, Hungary, 2003.

[8] Gielen R M A M, Jong L P D, Kerkvliet H M M. Electrooptical blood-spot detection in intact eggs. IEEE Transactions on Instrumentation \& Measurement, 1979; 28(3): 177-183.

[9] Xu H, Xu W, Chen H, Yang Y, Zhang A. Detection of blood spots in brown eggs based on spectroscopic techniques. Transactions of the CSAM, 2014; 45(2): 194-198.

[10] Huang M, Wan X, Zhang M, Zhu Q. Detection of insect-damaged vegetable soybeans using hyperspectral transmittance image. Journal of Food Engineering, 2013; 116(1): 45-49.

[11] Zhang W, Pan L, Tu S, Zhan G, Tu K. Non-destructive internal quality assessment of eggs using a synthesis of hyperspectral imaging and multivariate analysis. Journal of Food Engineering, 2015; 157: 41-48. 
[12] Bamelis F R, Ketelaere B D, Kemps B J, Mertens K, Decuypere E M, Baerdemaeker J G D. Non invasive methods for egg quality evaluation. 12th European Poultry Conference, Verona, Italy, 2006

[13] Nalbandov A V, Card L E. The problem of blood clots and meat spots in chicken eggs. Poultry Science, 1944; 23(3): 170-180.

[14] Chen M, Zhang L, Xu H. On-line detection of blood spot introduced into brown-shell eggs using visible absorbance spectroscopy. Biosystems Engineering, 2015; 131: 95-101.

[15] Xu W. A study on spectral features and real-time detection of blood spots in eggs. Hangzhou: Zhejiang University, 2013

[16] Qin J, Lu R. Detection of pits in tart cherries by hyperspectral transmission imaging. Proc Spie, 2005; 48(5): 1963-1970.

[17] Araújo M C U, Saldanha T C B, Galvão R K H, Yoneyama T, Chame H C, Visani V. The successive projections algorithm for variable selection in spectroscopic multicomponent analysis. Chemometrics \& Intelligent Laboratory Systems, 2001; 57(2): 65-73.

[18] Ye S, Wang D, Min S. Successive projections algorithm combined with uninformative variable elimination for spectral variable selection. Chemometrics \& Intelligent Laboratory Systems, 2008; 91(2): 194-199.

[19] Altman N S. An introduction to kernel and nearest-neighbor nonparametric regression. American Statistician, 1992; 46(3): 175-185.

[20] Hall P, Park B U, Samworth R J. Choice of neighbor order in nearest-neighbor classification. Annals of Statistics, 2008; 36(5): 2135-2152.

[21] Gold C, Sollich P. Model selection for support vector machine classification. Neurocomputing, 2002; 55(1): 221-249.

[22] Mercier G, Lennon M. Support vector machines for hyperspectral image classification with spectral-based kernels. Proceedings of 2003 IEEE International Symposium on Geoscience and Remote Sensing, IGARSS '03. 2003; 281: 288-290.

[23] Kemps B J, Bamelis F R, Ketelaere B D, Mertens K, Tona K, Decuypere E $\mathrm{M}$, et al. Visible transmission spectroscopy for the assessment of egg freshness. Journal of the Science of Food \& Agriculture, 2006; 86(9): 1399-1406. 